\title{
INFINITE DIMENSIONAL ROTATIONS AND LAPLACIANS IN TERMS OF WHITE NOISE CALCULUS
}

\author{
TAKEYUKI HIDA, NOBUAKI OBATA * AND KIMIAKI SAITÔ
}

\section{Introduction}

The theory of generalized white noise functionals (white noise calculus) initiated in [2] has been considerably developed in recent years, in particular, toward applications to quantum physics, see e.g. [5], [7] and references cited therein. On the other hand, since H. Yoshizawa [4], [23] discussed an infinite dimensional rotation group to broaden the scope of an investigation of Brownian motion, there have been some attempts to introduce an idea of group theory into the white noise calculus. For example, conformal invariance of Brownian motion with multidimensional parameter space [6], variational calculus of white noise functionals [14], characterization of the Lévy Laplacian [17] and so on.

The paper aims at establishing the fundamentals of infinite dimensional harmonic analysis within the framework of white noise calculus, namely, based on the calculus of differential operators $\partial_{t}$ and their dual operators $\partial_{t}^{*}$, where $t$ runs over a time parameter space $T$. We develop a general theory of operators acting on white noise functionals and, as a particular case, discuss infinite dimensional rotations and Laplacians in detail.

Let us now recall some notions of white noise calculus, for more precise information see Section 1 . Let $T$ be a topological space with a Borel measure $\nu$. We consider $T$ as a time parameter space including a multi-time parameter case where quantum field theory may be formulated. Let $E \subset L^{2}(T, \nu ; \mathbf{R})=H \subset E^{*}$ be a Gelfand triple constructed by means of a particular self-adjoint operator $A$. Let $\mu$ be the Gaussian measure on $E^{*}$ and put $\left(L^{2}\right)=L^{2}\left(E^{*}, \mu ; \mathrm{C}\right)$, which is canonically isomorphic to the Boson Fock space over $H_{\mathbf{C}}$. We then obtain a Gelfand triple $(E) \subset\left(L^{2}\right) \subset(E)^{*}$ by means of the second quantized operator $\Gamma(A)$. An element

Recieved September 26, 1991.

* Supported by the Alexander von Humboldt-Stiftung 
of $(E)$ (resp. $\left.(E)^{*}\right)$ is called a test (resp. generalized) white noise functional. For each $t \in T$ we define

$$
\partial_{t} \phi(x)=\lim _{\theta \rightarrow 0} \frac{\phi\left(x+\theta \delta_{t}\right)-\phi(x)}{\theta}, \quad \phi \in(E), \quad x \in E^{*},
$$

where $\delta_{t} \in E^{*}$ is the Dirac $\delta$-function at $t \in T$. Then $\partial_{t}$ becomes a continuous derivation on $(E)$ and $\partial_{t}^{*}$ a continuous linear operator on $(E)^{*}$. They correspond to the amnihilation and creation operators at a point $t \in T$, respectively, and satisfy the canonical commutation relation.

In this paper we establish an effective theory of continuous operators on $(E)$ expressed as superposition of $\partial_{t}$ and $\partial_{t}^{*}$ with normal ordering:

$(0-1) \Xi_{l, m}(\kappa)=\int_{T^{l+m}} \kappa\left(s_{1}, \ldots, s_{l}, t_{1}, \ldots, t_{m}\right) \partial_{s_{1}}^{*} \cdots \partial_{s_{l}}^{*} \partial_{t_{1}} \cdots \partial_{t m} d s_{1} \cdots d s_{l} d t_{1} \cdots d t_{m}$.

By means of duality argument we prove that (0-1) defines a continuous operator from $(E)$ into $(E)^{*}$ for any $\kappa \in\left(E_{\mathbf{C}}^{\otimes(l+m)}\right)^{*}$, namely, for any distribution $\kappa$ in $(l+m)$-variables (Theorem 2.2). The integral (0-1) is, therefore, understood in a generalized sense and $\Xi_{l, m}(\kappa)$ is called an integral kemel operator. Moreover, we have a criterion for checking when $\Xi_{l, m}(\kappa)$ defines a continuous operator on $(E)$ (Theorem 2.6). Since practically most important (usually unbounded) operators acting on $\left(L^{2}\right)$ are expressed as in the form of $(0-1)$, our theory will be effective to a systematic approach to the operator theory on a Fock space and further applications as well.

Let $O(E ; H)$ denote the infinite dimensional rotation group in the sense of Yoshizawa, namely, it is the group of orthogonal operators on $H$ which induce homeomorphisms of $E$. In other words, it is the automorphism group of the Gelfand triple $E \subset H \subset E^{*}$. Recalling that $x(t)=\partial_{t}+\partial_{t}^{*}$ is multiplication operator by a white noise coordinate (Proposition 4.4 ), we naturally come to a continuous operator from $(E)$ into $(E)^{*}$ :

$$
x(s) \partial_{t}-x(t) \partial_{s}=\partial_{s}^{*} \partial_{t}-\partial_{t}^{*} \partial_{s},
$$

which is a formal analogy of an infinitesimal generator of finite dimensional rotations. Using the general theory established in this paper, we investigate a definite role of (0-2). Namely, if $X$ is an infinitesimal generator of a regular one-parameter subgroup of $O(E ; H)$, there exists a skew-symmetric distribution $\kappa \in E \otimes E^{*}$ such that

$$
d \Gamma(X)=\int_{T \times T} \kappa(s, t)\left(\partial_{s}^{*} \partial_{t}-\partial_{t}^{*} \partial_{s}\right) d s d t
$$


where $d \Gamma$ is the differential representation (Theorem 4.3).

Infinite dimensional Laplacians have been so far discussed within the framework of white noise calculus, see e.g., [2], [10], [12], [19]. With our integral expression $(0-1)$ the Gross Laplacian and the number operator are respectively expressed as

$$
\begin{aligned}
\Delta_{G} & =\int_{T \times T} \tau(s, t) \partial_{s} \partial_{t} d s d t, \\
N & =\int_{T \times T} \tau(s, t) \partial_{s}^{*} \partial_{t} d s d t,
\end{aligned}
$$

where $\tau \in E \otimes E^{*}$ is the trace, namely, defined by $\langle\tau, \xi \otimes \eta\rangle=\langle\xi, \eta\rangle$, $\xi, \eta \in E$. By means of rotation-invariance we will characterize these operators among second order operators (Theorems 5.1 and 5.2).

The paper is organized as follows. Assembling some basic notions of white noise calculus in Section 1, we establish in Section 2 a general theory of integral kernel operators. In Section 3 we investigate some results on one-parameter groups of transformations in general. As a corollary we obtain the Taylor formula for white noise functionals. In Section 4 we prove that an infinitesimal generator of a one-parameter subgroup of the infinite dimensional rotation group is described in terms of $\partial_{s}^{*} \partial_{t}-\partial_{t}^{*} \partial_{s}$. Finally, in Section 5 we discuss infinite dimensional Laplacians in connection with their invariance under the infinite dimensional rotation group. The Appendix contains a few useful inequalities.

There have been a few approaches to the Lévy Laplacian [15] from the viewpoint of white noise calculus [8], [12], [13], [17]. We now have good hope that the Lévy Laplacian could also be characterized within our setup.

Acknowledgements. The authors welcome this opportunity to express their sincere gratitude to Professor J. Potthoff for interesting conversations which improved this paper considerably. The main part of the joint work was completed during the second named author's stay in Tübingen, Germany. He is very grateful to Professor H. Heyer and the Alexander von Humboldt Foundation for their warm hospitality.

\section{$\S 1$. Standard setup of white noise calculus}

We begin with some general notation. For a real vector space $\mathfrak{X}$ we denote its complexification by $\mathfrak{X}_{\mathbf{C}}$. If $\mathfrak{X}$ is a topological vector space, the dual space $\mathfrak{X}^{*}$ is always assumed to carry the strong dual topology. For two topological vector spaces 
$\mathfrak{X}$ and $\mathfrak{Y}$ let $\mathscr{L}(\mathfrak{X}, \mathfrak{Y})$ stand for the space of continuous linear operators from $\mathfrak{X}$ into $\mathfrak{Y}$. If $\mathfrak{X}$ and $\mathfrak{Y}$ are nuclear spaces, we denote simply by $\mathfrak{X} \otimes \mathfrak{Y}$ the completion of the algebraic tensor product $\mathfrak{X} \otimes$ alg $\mathfrak{Y}$ with respect to the $\pi$-topology, or equivalently the $\varepsilon$-topology, see e.g., [20]. If $H$ and $K$ are Hilbert spaces, we denote again by $H \otimes K$ the completed Hilbert space tensor product (hence $H \otimes K$ is again a Hilbert space). The somehow ambiguously used symbol, however, will cause no confusion in the context. If $\mathfrak{X}$ is a Hilbert space or a nuclear space, let $\mathfrak{X}^{\hat{\otimes} n} \subset \mathfrak{X}^{\otimes n}$ denote the closed subspace of symmetric tensor products. We also use the symbol $\left(\mathfrak{X}^{\otimes n}\right)_{\text {sym }}^{*}$ for the same meaning in case of dual spaces.

We then assemble some basic notions and notations of white noise calculus principally following [7], see also [1], [10], [11], [18] and [22].

Let $T$ be a topological space equipped with a Borel measure $d \nu(t)=d t$. Let $H=L^{2}(T, \nu ; \mathbf{R})$ be the real Hilbert space of square integrable functions on $T$. Its norm and inner product will be denoted by $|\cdot|_{0}$ and $\langle\cdot, \cdot\rangle$, respectively. Let $A$ be an operator on $H$ with domain $\operatorname{Dom}(A)$. We assume that $H$ admits a complete orthonormal basis $\left\{e_{j}\right\}_{j=0}^{\infty} \subset \operatorname{Dom}(A)$ such that

(A1) $A e_{j}=\lambda_{j} e_{j}$ for $\lambda_{j} \in \mathbf{R}$;

(A2) $1<\lambda_{0} \leq \lambda_{1} \leq \cdots \rightarrow \infty$;

(A3) $\sum_{j=0}^{\infty} \lambda_{j}^{-2}<\infty$.

Obviously, $A^{-1}$ is extended to an operator of Hilbert-Schmidt class. Put

$$
\rho=\lambda_{0}^{-1}=\left\|A^{-1}\right\|, \quad \delta=\left(\sum_{\jmath=0}^{\infty} \lambda_{j}^{-2}\right)^{1 / 2}=\left\|A^{-1}\right\|_{H S} .
$$

We also note the following apparent inequalities:

$$
0<\rho<1, \quad \rho<\delta .
$$

For $p \in \mathbf{R}$ let $E_{p}$ be the completion of $\operatorname{Dom}\left(A^{p}\right)$ with respect to the Hilbertian norm $|\xi|_{p}=\left|A^{p} \xi\right|_{0}, \xi \in \operatorname{Dom}\left(A^{p}\right)$, where $\operatorname{Dom}\left(A^{p}\right)=H$ for $p<0$. We then come to a chain of Hilbert spaces:

$$
\begin{array}{r}
\cdots \subset E_{p} \subset \cdots \subset E_{q} \subset \cdots \subset E_{0}=H \subset \cdots \subset E_{-q} \subset \cdots \subset E_{-p} \subset \cdots, \\
0 \leq q \leq p .
\end{array}
$$

Equipped with the Hilbertian norms $\left\{|\cdot|_{p}\right\}_{p \geq 0}$,

$$
E=\bigcap_{p \geq 0} E_{p}
$$

becomes a nuclear Fréchet space and its dual space is obtained as

$$
E^{*}=\underset{p \geq 0}{\bigcup} E_{-p}
$$


It is known that the strong dual topology of $E^{*}$ coincides with the inductive limit topology. The canonical bilinear form on $E^{*} \times E$ is denoted again by $\langle\cdot, \cdot\rangle$ and it is extended to a $\mathbf{C}$-bilinear form on $E_{\mathbf{C}}^{*} \times E_{\mathbf{C}}$. The symbols $|\cdot|_{p}$ and $\langle\cdot, \cdot\rangle$ are used for tensor products as well. For instance, it holds that

$$
|f|_{p} \leq \rho^{n}|f|_{p+1}, \quad f \in E_{\mathbf{C}}^{\otimes n}, \quad p \in \mathbf{R} .
$$

By construction $\xi \in E$ is a function on $T$ determined up to $\nu$-null functions. We then assume the following three conditions which are suggested by Kubo and Takenaka [10].

(H1) For every $\xi \in E$ there exists a unique continuous function on $T$ which coincides with $\xi$ up to $\nu$-null functions.

We agree then that $E$ consists of continuous functions.

(H2) For each $t \in T$ the evaluation map $\delta_{t}: \xi \mapsto \xi(t), \xi \in E$, is continuous, i.e., $\delta_{t} \in E^{*}$.

(H3) The map $t \mapsto \delta_{t} \in E^{*}, t \in T$, is continuous.

Under these conditions one may prove that any function in $E_{\mathbf{C}}^{\otimes n}, n=1,2, \ldots$, is a continuous function on $T^{n}$.

Let $\mu$ be the Gaussian measure on $E^{*}$ which is uniquely determined by the characteristic functional:

$$
\exp \left(-\frac{1}{2}|\xi|_{0}^{2}\right)=\int_{E^{*}} e^{\imath\langle x, \xi\rangle} d \mu(x), \quad \xi \in E
$$

We put $\left(L^{2}\right)=L^{2}\left(E^{*}, \mu ; \mathbf{C}\right)$ for simplicity and let $\|\cdot\|_{0}$ denote its norm. The Wiener-Itô decomposition theorem says $\left(L^{2}\right)$ is canonically isomorphic to the Fock space over $H_{\mathbf{C}}$ :

$$
\left(L^{2}\right) \cong \sum_{n=0}^{\infty} \oplus H_{\mathbf{C}}^{\hat{\otimes} n}
$$

If $\phi \in\left(L^{2}\right)$ corresponds to $\left(f_{n}\right)_{n=0}^{\infty}, f_{n} \in H_{\mathbf{C}}^{\widehat{\otimes} n}$, we have

$$
\|\phi\|_{0}^{2}=\sum_{n=0}^{\infty} n !\left|f_{n}\right|_{0}^{2}
$$

In that case we may write

$$
\phi(x)=\sum_{n=0}^{\infty}\left\langle: x^{\otimes n}:, f_{n}\right\rangle, \quad f_{n} \in H_{\mathbf{C}}^{\hat{\widehat{S}} n} .
$$

Here $: x^{\otimes n}: \in\left(E^{\otimes n}\right)_{\text {sym }}^{*}$ is defined inductively as follows: 


$$
\left\{\begin{array}{l}
: x^{\otimes 0}:=1 \\
: x^{\otimes 1}:=x \\
: x^{\otimes n}:=x \hat{\otimes}: x^{\otimes(n-1)}:-(n-1) \tau \hat{\otimes}: x^{\otimes(n-2)}:, \quad n \geq 2,
\end{array}\right.
$$

where $\tau \in(E \otimes E)_{\text {sym }}^{*}$ is defined by

$$
\tau=\sum_{j=0}^{\infty} e_{j} \otimes e_{j}
$$

Note that

$$
|\tau|_{-1}^{2}=\sum_{j=0}^{\infty} \lambda_{j}^{-4}<\delta^{2}
$$

In $(1-3)$ each $\left\langle: x^{\otimes n}:, f_{n}\right\rangle$ is defined only as $L^{2}$-function and the series is converges in $\left(L^{2}\right)$ according to $(1-2)$.

Through (1-2) and (1-3) we may introduce a second quantized operator. Let $\operatorname{Dom}(\Gamma(A))$ be the subspace of $\phi \in\left(L^{2}\right)$ given as in $(1-3)$ such that (i) $f_{n}=0$ except finitely many $n$; and (ii) $f_{n} \in \operatorname{Dom}(A) \bigotimes_{\mathrm{alg}} \cdots \bigotimes_{\mathrm{alg}} \operatorname{Dom}(A)(n$-times). Then for $\phi \in \operatorname{Dom}(\Gamma(A))$ we put

$$
(\Gamma(A) \phi)(x)=\sum_{n=0}^{\infty}\left\langle: x^{\otimes n}:, A^{\otimes n} f_{n}\right\rangle .
$$

As is easily seen, $\Gamma(A)$ satisfies (A1) and (A3) with replacing $A$ with $\Gamma(A)$. As for (A2) we observe that the smallest eigenvalue of $\Gamma(A)$ is exactly one. We then apply the method of constructing $E$ from $A$ to the white noise case.

Let $\left(E_{p}\right)$ be the completion of $\Gamma(A)^{p}$ with respect to the Hilbertian norm

$$
\|\phi\|_{p}^{2}=\left\|\Gamma(A)^{p} \phi\right\|_{0}^{2}=\sum_{n=0}^{\infty} n !\left|f_{n}\right|_{p}^{2},
$$

where $\phi$ and $\left(f_{n}\right)_{n=0}^{\infty}$ are related as in (1-3). Equipped with the norms $\left\{\|\cdot\|_{p}\right\}_{p} \geq 0$,

$$
(E)=\bigcap_{p \geq 0}\left(E_{p}\right)
$$

becomes a nuclear Fréchet space. Moreover, we note the following result due to Kubo and Yokoi [11], see also Yokoi [22].

Proposition 1.1. Let $\phi \in\left(L^{2}\right)$ be given as in $(1-3)$. Then $\phi \in(E)$ if and only if $f_{n} \in E_{\mathbf{C}}^{\hat{\otimes} n}$ for all $n=0,1,2, \ldots$ and $\sum_{n=0}^{\infty} n !\left|f_{n}\right|_{p}^{2}<\infty$ for all $p \geq 0$. In that case the right hand side of (1-3) converges pointwisely and becomes a unique con- 
tinuous function on $E^{*}$ which coincides with $\phi$ up to $\mu$-mull functions.

By the above fact we always regard $(E)$ as a space of continuous functions on $E^{*}$. Let $(E)^{*}$ be the dual space of $(E)$. An element in $(E)$ (resp. $(E)^{*}$ ) is called a test (resp. generalized) white noise functional. We denote by $\langle\langle\cdot, \cdot\rangle$ the canonical C-bilinear form on $(E)^{*} \times(E)$. When $T=\mathbf{R}$ and $A=1+t^{2}-$ $(d / d t)^{2},(E)$ and $(E)^{*}$ are often denoted by $(\&)$ and $(\&)^{*}$, respectively.

We now introduce a differential operator $\partial_{t}$ which plays a fundamental role in the white noise calculus. For $G_{m} \in\left(E_{\mathbf{C}}^{\otimes m}\right)^{*}$ and $f_{m+n} \in E_{\mathbf{C}}^{\hat{\otimes}(m+n)}$ we denote by $G_{m} \otimes_{m} f_{m+n} \in E_{\mathbf{C}}^{\hat{\otimes} n}$ uniquely determined by

$$
\left\langle G_{m} \otimes F_{n}, f_{m+n}\right\rangle=\left\langle F_{n}, G_{m} \otimes_{m} f_{m+n}\right\rangle, \quad F_{n} \in\left(E_{\mathbf{C}}^{\otimes n}\right)^{*} .
$$

For example, if $f_{n+1} \in E_{\mathbf{C}}^{\hat{\otimes}(n+1)}$, then

$$
\delta_{t} \otimes_{1} f_{n+1}\left(t_{1}, \ldots, t_{n}\right)=f_{n+1}\left(t, t_{1}, \ldots, t_{n}\right) .
$$

For $\phi \in(E)$ and $y \in E^{*}$ we put

$$
\left(D_{y} \phi\right)(x)=\sum_{n=1}^{\infty} n\left\langle: x^{\otimes(n-1)}:, y \otimes_{1} f_{n}\right\rangle,
$$

where $f_{n} \in E_{\mathbf{C}}^{\hat{\otimes} n}$ is given as in (1-3), see also Proposition 1.1. Since

$$
\left|y \otimes_{1} f_{n}\right|_{p} \leq \rho^{q(n-1)}|y|_{-(p+q)}\left|f_{n}\right|_{p+q}, \quad p, q \geq 0,
$$

which is easily verified by Fourier expansion or by Proposition A.1, we obtain

$$
\left\|D_{y} \phi\right\|_{p} \leq M_{1}|y|_{-(p+q)}\|\phi\|_{p+q} . \quad \phi \in(E) .
$$

where

$$
M_{1}=M_{1}(p, q)=\sup _{n \geq 0} \sqrt{n} \rho^{q(n-1)}<\infty . \quad q>0 .
$$

Therefore $D_{y}$ is a continuous linear operator on $(E)$. It is known that

$$
\left(D_{y} \phi\right)(x)=\lim _{\theta \rightarrow 0} \frac{\phi(x+\theta y)-\phi(x)}{\theta}, \quad \phi \in(E) .
$$

We now denote $D_{\delta_{t}}$ simply by $\partial_{t}$.

It is often convenient to use so-called exponential vectors. For $\xi \in \dot{E}_{\mathbf{C}}$ define $\phi_{\xi} \in(E)$ by

$$
\phi_{\xi}(x)=\sum_{n=0}^{\infty} \frac{1}{n !}\left\langle: x^{\otimes n}:, \xi^{\otimes n}\right\rangle .
$$


Then $\left\{\phi_{\xi} ; \xi \in E_{\mathbf{C}}\right\}$ spans a dense subspace of $(E)$. Note that

$$
\begin{gathered}
\left\langle\left\langle\phi_{\xi}, \phi_{\eta}\right\rangle\right\rangle=e^{\langle\xi, \eta\rangle}, \quad \xi, \eta \in E_{\mathbf{C}}, \\
D_{y} \phi_{\xi}=\langle y, \xi\rangle \phi_{\xi}, \quad y \in E^{*}, \quad \xi \in E_{\mathbf{C}} .
\end{gathered}
$$

In particular,

$$
\partial_{t} \phi_{\xi}=\xi(t) \phi_{\xi}, \quad t \in T, \quad \xi \in E_{\mathbf{C}} .
$$

These are easily verified.

\section{§ 2. Integral kernel operators}

Having introduced the differential operator $\partial_{t}$ in the previous section, we now develop a general theory of operators which are expressed as an integral of $\partial_{t}$ and $\partial_{t}^{*}$. We begin with

Lemma 2.1. For $\phi, \phi \in(E)$ we put

$$
\eta_{\phi, \psi}\left(s_{1}, \ldots, s_{l}, t_{1}, \ldots, t_{m}\right)=\left\langle\left\langle\partial_{s_{1}}^{*} \cdots \partial_{s_{l}}^{*} \partial_{t_{1}} \cdots \partial_{t_{m}} \phi, \phi\right\rangle\right\rangle .
$$

Then for any $p>0$ we have

$$
\left|\eta_{\phi, \phi}\right|_{p} \leq \rho^{-p}\left(l^{l} m^{m}\right)^{1 / 2}\left(\frac{\rho^{-p}}{-2 p e \log \rho}\right)^{(l+m) / 2}\|\phi\|_{p}\|\phi\|_{p} .
$$

In particular, $\eta_{\phi, \psi} \in E_{\mathbf{C}}^{\otimes(l+m)}$.

Proof. For simplicity we put $\eta=\eta_{\phi, \psi}$ and suppose

$$
\phi(x)=\sum_{n=0}^{\infty}\left\langle: x^{\otimes n}:, f_{n}\right\rangle \quad \text { and } \quad \phi(x)=\sum_{n=0}^{\infty}\left\langle: x^{\otimes n}:, g_{n}\right\rangle,
$$

where $f_{n}, g_{n} \in E_{\mathbf{C}}^{\hat{\otimes} n}$. Then, by a simple calculation we have

$$
\begin{aligned}
& \eta\left(s_{1}, \ldots, s_{l}, t_{1}, \ldots, t_{m}\right) \\
& =\left\langle\left\langle\partial_{t_{1}} \cdots \partial_{t m} \phi, \partial_{s_{l}} \cdots \partial_{s_{1}} \phi\right\rangle\right\rangle \\
& =\sum_{n=0}^{\infty} \frac{(m+n) !(l+n) !}{n !} \eta_{n}\left(s_{1}, \ldots, s_{l}, t_{1}, \ldots, t_{m}\right),
\end{aligned}
$$

where

$$
\eta_{n}\left(s_{1}, \ldots, s_{l}, t_{1}, \ldots, t_{m}\right)
$$




$$
\begin{aligned}
& =\left\langle\left(\delta_{t_{1}} \otimes \cdots \otimes \delta_{t m}\right) \otimes_{m} f_{m+n},\left(\delta_{s l} \otimes \cdots \otimes \delta_{s_{1}}\right) \otimes_{l} g_{l+n}\right\rangle \\
& =\int_{T^{n}} f_{m+n}\left(t_{m}, \ldots, t_{1}, u_{1}, \ldots, u_{n}\right) g_{l+n}\left(s_{1}, \ldots, s_{l}, u_{1}, \ldots, u_{n}\right) d u_{1} \cdots d u_{n} .
\end{aligned}
$$

Then, using the Schwarz inequality and Corollary A.2, we obtain

$$
\begin{aligned}
\left|\eta_{n}\right|_{p} & =\left|\left(A^{p}\right)^{\otimes(l+m)} \eta_{n}\right|_{0} \\
& \leq\left|\left(\left(A^{p}\right)^{\otimes m} \otimes I^{\otimes n}\right) f_{m+n}\right|_{0}\left|\left(\left(A^{p}\right)^{\otimes l} \otimes I^{\otimes n}\right) g_{l+n}\right|_{0} \\
& \leq \rho^{2 p n}\left|f_{m+n}\right|_{p}\left|g_{l+n}\right|_{p} .
\end{aligned}
$$

Hence from $(2-3)$ and $(2-4)$ we see that

$$
\begin{aligned}
|\eta|_{p} & \leq \sum_{n=0}^{\infty} \frac{(m+n) !(l+n) !}{n !}\left|\eta_{n}\right|_{p} \\
& \leq \sum_{n=0}^{\infty} \sqrt{\frac{(m+n) !}{n !}} \sqrt{\frac{(l+n) !}{n !}} \rho^{2 p n} \sqrt{(m+n) !}\left|f_{m+n}\right|_{p} \sqrt{(l+n) !}\left|g_{l+n}\right|_{p} \\
& \leq M_{2}\left(\sum_{n=0}^{\infty}(m+n) !\left|f_{m+n}\right|_{p}^{2}\right)^{1 / 2}\left(\sum_{n=0}^{\infty}(l+n) !\left|g_{l+n}\right|_{p}^{2}\right)^{1 / 2}
\end{aligned}
$$

where

$$
M_{2}=\sup _{n \geq 0} \sqrt{\frac{(m+n) !}{n !}} \sqrt{\frac{(l+n) !}{n !}} \rho^{2 p n}<\infty, \quad p>0 .
$$

Hence

$$
|\eta|_{p} \leq M_{2}\|\phi\|_{p}\|\phi\|_{p}
$$

and therefore, $\eta=\eta_{\phi, \psi} \in E_{\mathbf{C}}^{\otimes(l+m)}$. Finally, using repeatedly an elementary fact

$$
\max _{x \geq 0} x e^{-\beta x}=\frac{1}{\beta e} \cdot \quad \beta>0,
$$

we obtain

$$
M_{2} \leq \rho^{-p}\left(l^{l} m^{m}\right)^{1 / 2}\left(\frac{\rho^{-p}}{-2 p e \log \rho}\right)^{(l+m) / 2} .
$$

Then $(2-2)$ follows from $(2-5)$ and $(2-6)$ immediately.

Q.E.D.

THEOREN 2.2. For any $\kappa \in\left(E_{\mathbf{C}}^{\otimes(l+m)}\right)^{*}$ there exists a continuous linear operator $\Xi_{l, m}(\kappa) \in \mathscr{L}\left((E),(E)^{*}\right)$ such that

$$
\left\langle\left\langle\Xi_{l, m}(\kappa) \phi, \phi\right\rangle\right\rangle=\left\langle\kappa, \eta_{\phi, \phi}\right\rangle, \quad \phi, \phi \in(E),
$$


where $\eta_{\phi, \psi}$ is given in (2-1). Moreover, for any $p>0$ with $|\kappa|_{-p}<\infty$ it holds that

$$
\left\|\Xi_{l, m}(\kappa) \phi\right\|_{-p} \leq \rho^{-p}\left(l^{l} m^{m}\right)^{1 / 2}\left(\frac{\rho^{-p}}{-2 p e \log \rho}\right)^{(l+m) / 2}|\kappa|_{-p}\|\phi\|_{p} .
$$

Proof. Note first that $\phi, \phi \mapsto\left\langle\kappa, \eta_{\phi, \phi}\right\rangle, \phi, \phi \in(E)$, is a continuous bilinear form on $(E)$. In fact by Lemma 2.1 we have

$$
\begin{aligned}
& \left|\left\langle\kappa, \eta_{\phi, \phi}\right\rangle\right| \leq|\kappa|_{-p}\left|\eta_{\phi, \phi}\right|_{p} \\
& \quad \leq \rho^{-p}\left(l^{l} m^{m}\right)^{1 / 2}\left(\frac{\rho^{-p}}{-2 p e \log \rho}\right)^{(l+m) / 2}|\kappa|_{-p}\|\phi\|_{p}\|\phi\|_{p} .
\end{aligned}
$$

Therefore there is a continuous linear operator $\Xi_{l, m}(\kappa) \in \mathscr{L}\left((E),(E)^{*}\right)$ such that

$$
\left\langle\left\langle\Xi_{l, m}(\kappa) \phi, \phi\right\rangle\right\rangle=\left\langle\kappa, \eta_{\phi, \phi}\right\rangle, \quad \phi, \phi \in(E) .
$$

Hence (2-9) becomes

$$
\left|\left\langle\left\langle\Xi_{l, m}(\kappa) \phi, \phi\right\rangle\right\rangle\right| \leq \rho^{-p}\left(l^{l} m^{m}\right)^{1 / 2}\left(\frac{\rho^{-p}}{-2 p e \log \rho}\right)^{(l+m) / 2}|\kappa|_{-p}\|\phi\|_{p}\|\phi\|_{p}
$$

from which (2-8) follows immediately.

Q.E.D.

In view of $(2-7)$ we also employ a formal integral expression:

$$
\Xi_{l, m}(\kappa)=\int_{T^{l+m}} \kappa\left(s_{1}, \ldots, s_{l}, t_{1}, \ldots, t_{m}\right) \partial_{s_{1}}^{*} \cdots \partial_{s_{l}}^{*} \partial_{t_{1}} \cdots \partial_{t_{m}} d s_{1} \cdots d s_{l} d t_{1} \cdots d t_{m}
$$

This is called an integral kernel operator with kemel distribution $\kappa$.

Here we discuss some basic properties of integral kernel operators. We begin with the uniqueness of the kernel distribution. For $\kappa \in E_{\mathbf{C}}^{\otimes(l+m)}$ we difine $s_{l, m}(\kappa)$ $\in E_{\mathbf{C}}^{\otimes(l+m)}$ by the formula:

$$
\begin{aligned}
& \left\langle s_{l, m}(\kappa), \eta_{1} \otimes \cdots \eta_{l} \otimes \xi_{1} \otimes \cdots \xi_{m}\right\rangle \\
& =\frac{1}{l ! m !} \sum_{\substack{\sigma \in \subseteq_{l} \\
\tau \in \mathbb{S}_{m}}}\left\langle\kappa, \eta_{\sigma(1)} \otimes \cdots \eta_{\sigma(l)} \otimes \eta_{\tau(1)} \otimes \cdots \xi_{\tau(m)}\right\rangle,
\end{aligned}
$$

where $\xi_{i}, \eta_{j} \in E_{\mathbf{C}}$. Then a direct verification implies the following

PROPOSITION 2.3. For any $\kappa \in E_{\mathbf{C}}^{\otimes(l+m)}$ it holds that $\Xi_{l, m}\left(s_{l, m}(\kappa)\right)=\Xi_{l, m}(\kappa)$. If $\Xi_{l, m}(\kappa)=0$, then $s_{l, m}(\kappa)=0$. 
Recall that $(E)$ is a nuclear Fréchet space and hence reflexive. If $\Xi \in$ $\mathscr{L}\left((E),(E)^{*}\right)$, its adjoint $\Xi^{*}$ belongs to $\mathscr{L}\left((E),(E)^{*}\right)$ again. For the adjoint of an integral kernel operator we have

Proposition 2.4. Let $\kappa \in E_{\mathbf{C}}^{\otimes(l+m)}$. Then $\Xi_{l, m}(\kappa)^{*}=\Xi_{m, l}\left(t_{m, l}(\kappa)\right)$, where $t_{m, l}(\kappa)$ is defined by

$$
\left\langle t_{m, l}(\kappa), \eta \otimes \zeta\right\rangle=\langle\kappa, \zeta \otimes \eta\rangle, \quad \eta \in E_{\mathbf{C}}^{\otimes m}, \zeta \in E_{\mathbf{C}}^{\otimes l} .
$$

We next discuss when an integral kernel operator $\Xi_{l, m}(\kappa)$ belongs to $\mathscr{L}((E)$, $(E))$ which is a subclass of $\mathscr{L}\left((E),(E)^{*}\right)$. For that purpose we first recall the canonical isomorphism between $\mathscr{L}\left(E_{\mathbf{C}}^{\otimes m}, E_{\mathbf{C}}^{\otimes l}\right)$ and $\left(E_{\mathbf{C}}^{\otimes l}\right) \otimes\left(E_{\mathbf{C}}^{\otimes m}\right)^{*} \subset$ $\left(E_{\mathbf{C}}^{\otimes(l+m)}\right)^{*}$, where all the tensor products are topological as we agreed at the beginning of Section 1. If $\kappa \in\left(E_{\mathbf{C}}^{\otimes l}\right) \otimes\left(E_{\mathbf{C}}^{\otimes m}\right)^{*}$ and $K \in \mathscr{L}\left(E_{\mathbf{C}}^{\otimes m}, E_{\mathbf{C}}^{\otimes l}\right)$ are in correspondence, then

$$
\left\langle\kappa, \eta_{l} \otimes \zeta_{m}\right\rangle=\left\langle\eta_{l}, K \zeta_{m}\right\rangle, \quad \eta_{l} \in E_{\mathbf{C}}^{\otimes l}, \zeta_{m} \in E_{\mathbf{C}}^{\otimes m},
$$

for further details, see e.g., [20: Chap.50]. The next result is easily derived.

LeMma 2.5 For $\kappa \in\left(E_{\mathbf{C}}^{\otimes(l+m)}\right)^{*}$ the following two conditions are equivalent:

(i) $\kappa \in\left(E_{\mathbf{C}}^{\otimes l}\right) \otimes\left(E_{\mathbf{C}}^{\otimes m}\right)^{*}$;

(ii) for any $p \geq 0$ there exist $C \geq 0$ and $q \geq 0$ such that $|\langle\kappa, \eta \otimes \zeta\rangle| \leq$ $C|\eta|_{-p}|\zeta|_{p+q}$ for $\eta \in E_{\mathbf{C}}^{\otimes l}$ and $\zeta \in E_{\mathbf{C}}^{\otimes m}$.

THEOREM 2.6. Let $\kappa \in\left(E_{\mathbf{C}}^{\otimes(l+m)}\right)^{*}$. Then $\Xi_{l, m}(\kappa) \in \mathscr{L}((E),(E))$ if and only if $\kappa \in\left(E_{\mathbf{C}}^{\otimes l}\right) \otimes\left(E_{\mathbf{C}}^{\otimes m}\right)^{*}$.

Proof. First suppose that $\kappa \in\left(E_{\mathbf{C}}^{\otimes l}\right) \otimes\left(E_{\mathbf{C}}^{\otimes m}\right)^{*}$ and let $K \in \mathscr{L}\left(E_{\mathbf{C}}^{\otimes m}, E_{\mathbf{C}}^{\otimes l}\right)$ be the corresponding operator determined as in $(2-10)$. Let $\phi, \phi \in(E)$ and we keep to the notations in the proof of Lemma 2.1. We then observe from $(2-3)$ and $(2-4)$ that

$$
\left\langle\left\langle\Xi_{l, m}(\kappa) \phi, \phi\right\rangle\right\rangle=\sum_{n=0}^{\infty} \frac{(m+n) !(l+n) !}{n !}\left\langle\kappa, \eta_{n}\right\rangle .
$$

Then, by $(2-10)$ we have

$$
\left\langle\left\langle\Xi_{l, m}(\kappa) \phi, \phi\right\rangle\right\rangle=\sum_{n=0}^{\infty} \frac{(m+n) !(l+n) !}{n !}\left\langle g_{l+n},\left(K \otimes I^{\otimes n}\right) f_{m+n}\right\rangle .
$$

Since $K$ is continuous, for a given $p \geq 0$ we may find $q \geq 0$ and $C=C(p, q)$ $\geq 0$ such that $|K \eta|_{p} \leq C|\eta|_{p+q}, \eta \in E_{\mathbf{C}}^{\otimes m}$. Then, applying Proposition A.1, we 
obtain

$$
\begin{aligned}
\left|\left\langle g_{l+n},\left(K \otimes I^{\otimes n}\right) f_{m+n}\right\rangle\right| \leq & \left|g_{l+n}\right|_{-p}\left|\left(K \otimes I^{\otimes n}\right) f_{m+n}\right|_{p} \\
& \leq C \rho^{q n}\left|f_{m+n}\right|_{p+q}\left|g_{l+n}\right|_{-p} .
\end{aligned}
$$

In view of $(2-11)$ and $(2-12)$ we obtain

$$
\begin{aligned}
\left|\left\langle\Xi_{l, m}(\kappa) \phi, \phi\right\rangle\right| & \leq \sum_{n=0}^{\infty} \frac{(m+n) !(l+n) !}{n !} C \rho^{q n}\left|f_{m+n}\right|_{p+q}\left|g_{l+n}\right|_{-p} \\
& \leq M_{3} \sum_{n=0}^{\infty} \sqrt{(m+n) !}\left|f_{m+n}\right|_{p+q} \sqrt{(l+n) !}\left|g_{l+n}\right|_{-p} \\
& \leq M_{3}\left(\sum_{n=0}^{\infty}(m+n) !\left|f_{m+n}\right|_{p+q}^{2}\right)^{1 / 2}\left(\sum_{n=0}^{\infty}(l+n) !\left|g_{l+n}\right|_{-p}^{2}\right)^{1 / 2} \\
& \leq M_{3}\|\phi\|_{p+q}\|\phi\|_{-p},
\end{aligned}
$$

where

$$
M_{3}=M_{3}(l, m, p, q)=\sup _{n \geq 0} \sqrt{\frac{(m+n) !}{n !}} \sqrt{\frac{(l+n) !}{n !}} C \rho^{q n}<\infty
$$

for $q>0$. Consequently,

$$
\left\|\Xi_{l, m}(\kappa) \phi\right\|_{p} \leq M_{3}\|\phi\|_{p+q} .
$$

This means that $\Xi_{l, m}(\kappa)$ is a continuous linear operator on $(E)$.

Conversely, suppose that $\Xi_{l, m}(\kappa) \in \mathscr{L}((E),(E))$. Then, for any $p \geq 0$ there exist $C \geq 0$ and $q \geq 0$ such that

$$
\left\|\Xi_{l, m}(\kappa) \phi\right\|_{p} \leq C\|\phi\|_{p+q} . \quad \phi \in(E) .
$$

Now consider

$$
\phi(x)=\left\langle: x^{\otimes m}:, \zeta\right\rangle, \quad \phi(x)=\left\langle: x^{\otimes l}:, \eta\right\rangle,
$$

where $\eta \in E_{\mathbf{C}}^{\otimes l}$ and $\zeta \in E_{\mathbf{C}}^{\otimes m}$. By definition we have

$$
\left\langle\left\langle\Xi_{l, m}(\kappa) \phi, \phi\right\rangle=l ! m !\langle\kappa, \eta \otimes \zeta\rangle,\right.
$$

and therefore,

$$
|\langle\kappa, \eta \otimes \zeta\rangle| \leq \frac{C}{l ! m !}\|\phi\|_{p+q}\|\phi\|_{-p}=\frac{C}{\sqrt{l ! m !}}|\eta|_{-p}|\zeta|_{p+q} .
$$

It then follows from Lemma 2.5 that $\kappa \in\left(E_{\mathbf{C}}^{\otimes l}\right) \otimes\left(E_{\mathbf{C}}^{\otimes m}\right)^{*}$.

Q.E.D.

The action of $\Xi_{l, m}(\kappa)$ on exponential vectors (see (1-9)) is given explicitly. 
Proposition 2.7. (1) Let $\kappa \in\left(E_{\mathbf{C}}^{\otimes(l+m)}\right)^{*}$. Then

$$
\left\langle\left\langle\Xi_{l, m}(\kappa) \phi_{\xi}, \phi_{\eta}\right\rangle\right\rangle=\left\langle\kappa, \eta^{\otimes l} \otimes \xi^{\otimes m}\right\rangle e^{\langle\xi, \eta\rangle}, \quad \xi, \eta \in E_{\mathbf{C}} .
$$

) Let $\kappa \in\left(E_{\mathbf{C}}^{\otimes l}\right) \otimes\left(E_{\mathbf{C}}^{\otimes m}\right)^{*}$ and $K \in \mathscr{L}\left(E_{\mathbf{C}}^{\otimes m}, E_{\mathbf{C}}^{\otimes l}\right)$ the corresponding operator demined as in (2-10). Then

$$
\Xi_{l, m}(\kappa) \phi_{\xi}(x)=\sum_{n=0}^{\infty} \frac{1}{n !}\left\langle: x^{\otimes(l+n)}:,\left(K \xi^{\otimes m}\right) \otimes \xi^{\otimes n}\right\rangle .
$$

) For $y \in E^{*}$ it holds that

$$
D_{y}=\Xi_{0,1}(y)=\int_{T} y(t) \partial_{t} d t
$$

Proof. (1) We need only to combine (2-7), (1-10) and (1-12).

$$
\begin{aligned}
\left\langle\left\langle\Xi_{l, m}(\kappa) \phi_{\xi}, \phi_{\eta}\right\rangle\right\rangle & =\left\langle\kappa,\left\langle\left\langle\partial_{t_{1}} \cdots \partial_{t m} \phi_{\xi}, \partial_{s_{l}} \cdots \partial_{s_{1}} \phi_{\eta}\right\rangle\right\rangle\right\rangle \\
& =\left\langle\kappa, \eta\left(s_{1}\right) \cdots \eta\left(s_{l}\right) \xi\left(t_{1}\right) \cdots \xi\left(t_{m}\right)\right\rangle\left\langle\left\langle\phi_{\xi}, \phi_{\eta}\right\rangle\right\rangle \\
& =\left\langle\kappa, \eta^{\otimes l} \otimes \xi^{\otimes m}\right\rangle e^{\langle\xi, \eta\rangle} .
\end{aligned}
$$

) Let $\phi(x)$ be the right hand side of the identity to be verified. By definition,

$$
\begin{aligned}
\left.\left\langle\psi, \phi_{\eta}\right\rangle\right\rangle & =\sum_{n=0}^{\infty}(l+n) !\left\langle\frac{1}{n !}\left(K \xi^{\otimes m}\right) \otimes \xi^{\otimes n}, \frac{1}{(l+n) !} \eta^{\otimes(l+n)}\right\rangle \\
& =\sum_{n=0}^{\infty} \frac{1}{n !}\left\langle K \xi^{\otimes m}, \eta^{\otimes l}\right\rangle\langle\xi, \eta\rangle^{n} \\
& =\left\langle\kappa, \eta^{\otimes l} \otimes \xi^{\otimes m}\right\rangle e^{\langle\xi, \eta\rangle} .
\end{aligned}
$$

view of (1) we conclude that $\Xi_{l, m}(\kappa) \phi_{\xi}=\phi$.

) It follows from (2) and (1-11) that

$$
\Xi_{0,1}(y) \phi_{\xi}=\sum_{n=0}^{\infty} \frac{1}{n !}\left\langle: x^{\otimes n}:\langle y, \xi\rangle \xi^{\otimes n}\right\rangle=\langle y, \xi\rangle \phi_{\xi}=D_{y} \phi_{\xi} .
$$

lerefore $\Xi_{0,1}(y)=D_{y}$.

Q.E.D.

Remark. During the proof of Theorem 2.6 we have observed the following sult: Let $K \in \mathscr{L}\left(E_{\mathbf{C}}^{\otimes m}, E_{\mathbf{C}}^{\otimes l}\right)$ and $\kappa \in\left(E_{\mathbf{C}}^{\otimes l}\right) \otimes\left(E_{\mathbf{C}}^{\otimes m}\right)^{*}$ be related as in $(2-10)$. $|K \eta|_{p} \leq C|\eta|_{p+q}$ with some $p \geq 0, q>0$ and $C \geq 0$, then $\left\|\Xi_{l, m}(\kappa) \phi\right\|_{p} \leq$ ' $\|\phi\|_{p+q}$ with some $M \geq 0$. Specializing this result for $\kappa=y \in E^{*}$ and $\Xi_{0,1}(y)$ $D_{y}$, we obtain a result due to Yan [21]: If $|y|_{-p}<\infty$ and $q<p$, then $\left\|D_{y} \phi\right\|_{q}$ $C\|\phi\|_{p}$. This is, of course, the same as $(1-7)$.

Combining Proposition 2.4 and Theorem 2.6, we come to the following 
THEOREM 2.8. If $\kappa \in\left(E_{\mathbf{C}}^{\otimes l}\right) \otimes\left(E_{\mathbf{C}}^{\otimes m}\right)^{*}$, then

$$
\Xi_{m, l}\left(t_{m, l}(\kappa)\right)=\int_{T^{m+l}} \kappa\left(s_{1}, \ldots, s_{l}, t_{1}, \ldots, t_{m}\right) \partial_{t_{1}}^{*} \cdots \partial_{t m}^{*} \partial_{s_{1}} \cdots \partial_{s_{l}} d t_{1} \cdots d t_{m} d s_{1} \cdots d s_{l}
$$

is extended to a continuous linear operator from $(E) *$ into itself.

If $\Xi \in \mathscr{L}\left((E),(E)^{*}\right)$ can be extended to a continuous linear operator from $(E)^{*}$ into itself, the extension is denoted by $\tilde{E}$. For example, $D_{\xi}$ is extended to $\tilde{D}_{\xi} \in \mathscr{L}\left((E)^{*},(E)^{*}\right)$ whenever $\xi \in E$.

\section{§ 3. One-parameter groups of transformations in general}

In this section $\mathfrak{X}$ denotes a barreled Hausdorff locally convex vector space with defining seminorms $\left\{\|\cdot\|_{\alpha}\right\}_{\alpha \in A}$. Recall that every Fréchet space is such a space, for further information see [20]. Let $G L(\mathfrak{X})$ be the group of linear homeomorphisms from $\mathfrak{X}$ onto itself. We put $\mathscr{L}(\mathfrak{X})=\mathscr{L}(\mathfrak{X}, \mathfrak{X})$ for simplicity. Obviously, $G L(\mathfrak{X}) \subset \mathscr{L}(\mathfrak{X})$.

A one-parameter subgroup $\left\{G_{\theta}\right\}_{\theta \in \mathbf{R}} \subset G L(\mathfrak{X})$ is called differentiable if $\lim _{\theta \rightarrow 0}\left(G_{\theta} \xi-\xi\right) / \theta$ converges in $\mathfrak{X}$ for any $\xi \in \mathfrak{X}$. If $\left\{G_{\theta}\right\}_{\theta \in \mathbf{R}}$ is differentiable, a linear operator $X$ from $\mathfrak{X}$ into itself is defined by

$$
X \xi=\lim _{\theta \rightarrow 0} \frac{G_{\theta} \xi-\xi}{\theta}, \quad \xi \in \mathfrak{X} .
$$

As usual, this operator $X$ is called the infinitesimal generator of the differentiable one-parameter subgroup $\left\{G_{\theta}\right\}_{\theta \in \mathbf{R}} \subset G L(\mathfrak{X})$. The next result is immediate from the Banach-Steinhaus theorem, e.g., see [20: Theorem 33.1].

Proposition 3.1. Let $\left\{G_{\theta}\right\}_{\theta \in \mathbf{R}} \subset G L(\mathfrak{X})$ be a differentiable one-parameter subgroup. Then its infinitesimal generator $X$ is always continuous, i.e., $X \in \mathscr{L}(\mathfrak{X})$. Moreover, the convergence (3-1) is uniform on every compact subset of $\mathfrak{X}$, namely,

$$
\lim _{\theta \rightarrow 0} \sup _{\xi \in K}\left\|\frac{G_{\theta} \xi-\xi}{\theta}-X \xi\right\|_{\alpha}=0
$$

for any $\alpha \in A$ and any compact subset $K \subset \mathfrak{X}$.

Remark. When $\mathfrak{X}$ is a nuclear Fréchet space, every bounded closed subset of $\mathfrak{X}$ is compact. Therefore, in that case the topology of $\mathscr{L}(\mathfrak{X})$ induced from uniform convergence on every compact subset of $\mathfrak{X}$ is equivalent to that of uniform con- 
vergence on every bounded subset of $\mathfrak{X}$.

A differetiable one-parameter subgroup is uniquely determined by its infinitesimal generator, namely, we have

Proposition 3.2. Let $\left\{G_{\theta}\right\}_{\theta \in \mathbf{R}}$ and $\left\{H_{\theta}\right\}_{\theta \in \mathbf{R}}$ be two differentiable one-parameter subgroups of $G L(\mathfrak{X})$ with the same infinitesimal generator $X$. Then $G_{\theta}=H_{\theta}$ for all $\theta \in \mathbf{R}$.

For the proof we need two straightforward results.

Lemma 3.3. Let $\left\{G_{\theta}\right\}_{\theta \in \mathbf{R}} \subset G L(\mathfrak{X})$ be a differentiable one-parameter subgroup with infinitesimal generator $X$. Then for any $\theta \in \mathbf{R}$ and any $\xi \in \mathfrak{X}$ we have

$$
G_{\theta} X \xi=X G_{\theta} \xi=\lim _{\varepsilon \rightarrow 0} \frac{G_{\theta+\varepsilon} \xi-G_{\theta} \xi}{\varepsilon} .
$$

Moreover, the convergence is uniform on every compact subset in $\mathfrak{X}$.

Lemma 3.4. Let $\left\{G_{\theta}\right\}_{\theta \in \mathbf{R}} \subset G L(\mathfrak{X})$ be a differentiable one-parameter subgroup. Then,

$$
\lim _{\varepsilon \rightarrow 0} \sup _{\xi \in K}\left\|G_{\theta+\varepsilon} \xi-G_{\theta} \xi\right\|_{\alpha}=0
$$

for any $\alpha \in A$ and any compact subset $K \subset \mathfrak{X}$.

Proof of Proposition 3.2. Let $\xi_{0} \in \mathfrak{X}$ be arbitrarily fixed. For simplicity we put $\xi(\theta)=H_{-\theta} \xi_{0}$. It becomes a differentiable curve in $\mathfrak{X}$ and from Lemma 3.3 we see that

$$
\frac{d}{d \theta} \xi(\theta)=-X H_{-\theta} \xi_{0}=-X \xi(\theta) .
$$

Furthermore, $\left\{G_{\theta} \xi(\theta)\right\}_{\theta \in \mathbf{R}}$ is also a differentiable curve in $\mathfrak{X}$. In fact, a simple verification with Lemma 3.4 leads us to the following

$$
\frac{d}{d \theta}\left(G_{\theta} \xi(\theta)\right)=G_{\theta}(-X \xi(\theta))+X G_{\theta} \xi(\theta)=0, \quad \theta \in \mathbf{R}
$$

Namely, $G_{\theta} \xi(\theta)=G_{0} \xi(0)=\xi_{0}$ for all $\theta \in \mathbf{R}$, and therefore $G_{\theta} \xi_{0}=H_{\theta} \xi_{0}$. Since $\xi_{0} \in \mathfrak{X}$ is arbitrary, we conclude that $G_{\theta}=H_{\theta}$.

Q.E.D. 
In general, not every $X \in \mathscr{L}(\mathfrak{X})$ can be an infinitesimal generator of a differentiable one-parameter subgroup of $G L(\mathfrak{X})$. We give here a sufficient condition.

Proposition 3.5. Let $X \in \mathscr{L}(\mathfrak{X})$ and assume that there exists $r>0$ such that $\left\{(r X)^{n} / n !\right\}_{n=0}^{\infty}$ is equicontinuous, namely, for every $\alpha \in A$ there exist $C=C(\alpha) \geq 0$ and $\beta=\beta(\alpha) \in A$ such that

$$
\sup _{n \geq 0} \frac{1}{n !}\left\|(r X)^{n} \xi\right\|_{\alpha} \leq C\|\xi\|_{\beta}, \quad \xi \in \mathfrak{X} .
$$

Then there exists a differentiable one-parameter subgroup $\left\{G_{\theta}\right\}_{\theta \in \mathbf{R}} \subset G L(\mathfrak{X})$ with infinitesimal generator $X$.

Proof. By assumpiton, the series

$$
G_{\theta} \xi=\sum_{n=0}^{\infty} \frac{\theta^{n}}{n !} X^{n} \xi, \quad \xi \in \mathfrak{X}, \quad|\theta|<r,
$$

is convergent in $\mathfrak{X}$ and $\left\|G_{\theta} \xi\right\|_{\alpha} \leq C(1-|\theta| / r)^{-1}\|\xi\|_{\beta}$, namely, $G_{\theta} \in \mathscr{L}(\mathfrak{X})$ for $|\theta|<r$. Furthermore, $G_{0}=I$ and $G_{\theta_{1}+\theta_{2}}=G_{\theta_{1}} G_{\theta_{2}}$ whenever $\left|\theta_{1}\right|,\left|\theta_{2}\right|$, $\left|\theta_{1}+\theta_{2}\right|<r$. We now define $G_{\theta}$ for all $\theta \in \mathbf{R}$. For a given $\theta \in \mathbf{R}$ choose a positive integer $n$ such that $|\theta / n|<r$ and put $G_{\theta}=\left(G_{\theta / n}\right)^{n}$. As is easily seen, this definition is independent of the choice of $n$, and therefore $G_{\theta_{1}+\theta_{2}}=G_{\theta_{1}} G_{\theta_{2}}$ for all $\theta_{1}, \theta_{2} \in \mathbf{R}$. Since

$$
\begin{aligned}
\left\|\frac{G_{\theta} \xi-\xi}{\theta}-X \xi\right\|_{\alpha} & \leq \sum_{n=2}^{\infty} \frac{|\theta|^{n-1}}{n !}\left\|X^{n \xi}\right\|_{\alpha} \\
& \leq|\theta| C r^{-2}\left(1-\frac{|\theta|}{r}\right)^{-1}\|\xi\|_{\beta}, \quad|\theta|<r,
\end{aligned}
$$

$\left\{G_{\theta}\right\}_{\theta \in \mathbf{R}}$ is a differentiable one-parameter subgroup of $G L(\mathfrak{X})$ with infinitesimal generator $X$.

Q.E.D.

During the above proof a somewhat stronger property of $\left\{G_{\theta}\right\}_{\theta \in \mathbf{R}}$ has been observed, cf. (3-2): for any $\alpha \in A$ there exists $\beta \in A$ such that

$$
\lim \sup _{\theta \rightarrow 0}\left\|\frac{G_{\theta} \xi-\xi}{\theta}-X \xi\right\|_{\beta} \leq 1=0 .
$$

If a differentiable one-parameter subgroup has this property, we call it regular. This notion will be useful when we consider the second quantization of the action of the infinite dimensional rotation group, see the next section. 
Remark. For $X \in \mathscr{L}(\mathfrak{X})$ consider the following condition: for any $\alpha \in A$ there exist constant numbers $C \geq 0,0 \leq \delta<1$ and $\beta \in A$ such that

$$
\left\|X^{n} \xi\right\|_{\alpha} \leq C(n !)^{\delta}\|\xi\|_{\beta}, \quad \xi \in \mathfrak{X} .
$$

This condition is apparently stronger than that in Proposition 3.5. Under this condition $G_{\theta}$ is defined by (3-3) for all $\theta \in \mathbf{R}$.

We end this section with an example. For $y \in E^{*}$ we defined a differential operator $D_{y}$ on $(E)$ by the formula (1-6). In a similar way as in (1-7), for $p \geq 0$ and $q>0$ we obtain

$$
\left\|D_{y}^{n} \phi\right\|_{p} \leq C(n)|y|_{-(p+q)}^{n}\|\phi\|_{p+q},
$$

where

$$
C(n)=\sup _{k \geq 0} \sqrt{\frac{(k+n) !}{k !}} \rho^{q k}<\infty .
$$

By a simple calculation $C(n) \leq n^{n / 2}$ whenever $q \geq(-2 \log \rho)^{-1}$. Taking $q>0$ large enough to hold $|y|_{-(p+q)}<\infty$ too, we obtain

$$
\left\|D_{y}^{n} \phi\right\|_{p} \leq n^{n / 2}|y|_{-(p+q)}^{n}\|\phi\|_{p+q} \leq C(n !)^{\delta}\|\phi\|_{p+q}
$$

for some $C \geq 0$ and $0 \leq \delta<1$. (In fact, by the Stirling formula we may take any $\delta$ with $1 / 2<\delta<1$.) Therefore $D_{y}$ is an infinitesimal generator of a regular one-parameter subgroup of $G L((E))$. As is expected from (1-8), the one-parameter subgroup is given by $\left\{T_{\theta y}\right\}_{\theta \in \mathbf{R}}$, where

$$
\left(T_{\theta y} \phi\right)(x)=\phi(x+\theta y), \quad \phi \in(E) .
$$

Furthermore, as a direct consequence of the above Remark, we obtain the Taylor formula for white noise functionals due to Potthoff and Yan [18].

THeOREm 3.6. For any $y \in E^{*}$ it holds that

$$
T_{y} \phi=\sum_{n=0}^{\infty} \frac{1}{n !} D_{y}^{n} \phi, \quad \phi \in(E),
$$

where the series is convergent in $(E)$.

\section{$\S 4$. Infinite dimensional rotations}

For $X \in \mathscr{L}(E)$ we introduce two operators $\Gamma(X)$ and $d \Gamma(X)$ on $(E)$. Let $\phi \in(E)$ be given by 


$$
\phi(x)=\sum_{n=0}^{\infty}\left\langle: x^{\otimes n}:, f_{n}\right\rangle, \quad x \in E^{*}, \quad f_{n} \in E_{\mathbf{C}}^{\otimes n}
$$

as before, see Proposition 1.1. Then we define

$$
\begin{gathered}
(\Gamma(X) \phi)(x)=\sum_{n=0}^{\infty}\left\langle: x^{\otimes n}:, X^{\otimes n} f_{n}\right\rangle, \\
(d \Gamma(X) \phi)(x)=\sum_{n=0}^{\infty} n\left\langle: x^{\otimes n}:,\left(X \otimes I^{\otimes(n-1)}\right) f_{n}\right\rangle .
\end{gathered}
$$

It is not difficult to prove that both $\Gamma(X)$ and $d \Gamma(X)$ belong to $\mathscr{L}((E))$. However, it is not clear whether $\left\{\Gamma\left(G_{\theta}\right)\right\}$ becomes a differentiable one-parameter subgroup of $G L((E))$ for any differentiable one-parameter subgroup $\left\{G_{\theta}\right\}_{\theta \in \mathbf{R}}$ of $G L(E)$. In this connection we have

THEOREM 4.1. Let $\left\{G_{\theta}\right\}_{\theta \in \mathbf{R}}$ be a regular one-parameter subgroup of $G L(E)$ with infinitesimal generator $X$. Then, $\left\{\Gamma\left(G_{\theta}\right)\right\}_{\theta \in \mathbf{R}}$ is a regular one-parameter subgroup of $G L((E))$ with infinitesimal generator $d \Gamma(X)$.

For the proof we need some inequalities. Suppose that $p \geq 0$ is given. From the regularity of $\left\{G_{\theta}\right\}_{\theta \in \mathbf{R}}$ there exists $q \geq 0$ such that

$$
\lim _{\theta \rightarrow 0} \sup _{|\xi|_{p+q} \leq 1}\left|\frac{G_{\theta} \xi-\xi}{\theta}-X \xi\right|_{p}=0 .
$$

Moreover, we may assume that with some $C \geq 0$,

$$
\begin{aligned}
& |X \xi|_{p} \leq C|\xi|_{p+q}, \\
& \delta \rho^{q+1}+2 \rho^{q+2}<1 .
\end{aligned}
$$

Suppose next $\varepsilon>0$ is given. In view of $(4-4)$ there exists $\theta_{0}>0$ such that

$$
\left|\frac{G_{\theta} \xi-\xi}{\theta}-X \xi\right|_{p} \leq \varepsilon|\xi|_{p+q}, \quad|\theta|<\theta_{0} .
$$

Furthermore, by (4-6) we may assume

$$
\delta \rho(\varepsilon+C) \theta_{0}+\delta \rho^{q+1}+2 \rho^{q+2}<1 .
$$

We then obtain

$$
\left|G_{\theta} \xi-\xi\right|_{p} \leq(\varepsilon+C)|\theta||\xi|_{p+q}
$$

and 


$$
\left|G_{\theta} \xi\right|_{p} \leq M_{4}|\xi|_{p+q},
$$

where $|\theta|<\theta_{0}$ and $M_{4}=(\varepsilon+C) \theta_{0}+\rho^{q}$.

Proof of Theorem 4.1. For simplicity we put

$$
\left\{\begin{array}{l}
\gamma_{n}(X)=\sum_{k=0}^{n-1} I^{\otimes k} \otimes X \otimes I^{\otimes(n-1-k)}, \quad n \geq 1, \\
\gamma_{0}(X)=0 .
\end{array}\right.
$$

By a simple calculation we have

$$
\begin{aligned}
\frac{G_{\theta}^{\otimes n}-I^{\otimes n}}{\theta}-\gamma_{n}(X)=\sum_{k=0}^{n-1} & \left\{I^{\otimes k} \otimes\left(\frac{G_{\theta}-I}{\theta}-X\right) \otimes G_{\theta}^{\otimes(n-1-k)}\right\} \\
& +\sum_{k=0}^{n-1}\left\{I^{\otimes k} \otimes X \otimes\left(G_{\theta}^{\otimes(n-1-k)}-I^{\otimes(n-1-k)}\right)\right\}
\end{aligned}
$$

and therefore, for $f_{n} \in E_{\mathbf{C}}^{\otimes n}$ it holds that

$$
\begin{aligned}
\left|\frac{G_{\theta}^{\otimes n} f_{n}-f_{n}}{\theta}-\gamma_{n}(X) f_{n}\right|_{p} \\
\leq \sum_{k=0}^{n-1}\left|\left(I^{\otimes k} \otimes\left(\frac{G_{\theta}-I}{\theta}-X\right) \otimes G_{\theta}^{\otimes(n-1-k)}\right) f_{n}\right|_{p} \\
\quad+\sum_{k=0}^{n-1}\left|\left(I^{\otimes k} \otimes X \otimes\left(G_{\theta}^{\otimes(n-1-k)}-I^{\otimes(n-1-k)}\right)\right) f_{n}\right|_{p} .
\end{aligned}
$$

In view of (4-7), (4-10) and Corollary A.4, we obtain

$$
\begin{aligned}
& \left|\left(I^{\otimes k} \otimes\left(\frac{G_{\theta}-I}{\theta}-X\right) \otimes G_{\theta}^{\otimes(n-1-k)}\right) f_{n}\right|_{p} \\
& \quad \leq \varepsilon M_{4}^{n-1-k} \rho^{1+(q+1) k} \delta^{n-1-k}\left|f_{n}\right|_{p+q+1} \\
& \quad=\varepsilon \rho\left(\delta M_{4}\right)^{n-1-k} \rho^{(q+1) k}\left|f_{n}\right|_{p+q+1} .
\end{aligned}
$$

Hence,

$$
\begin{aligned}
\sum_{k=0}^{n-1} \mid\left(I^{\otimes k}\right. & \left.\otimes\left(\frac{G_{\theta}-I}{\theta}-X\right) \otimes G_{\theta}^{\otimes(n-1-k)}\right)\left.f_{n}\right|_{p} \\
& \leq \varepsilon \rho M_{5}^{n-1}\left|f_{n}\right|_{p+q+1} \\
& \leq \varepsilon \rho^{2}\left(\rho M_{5}\right)^{n-1}\left|f_{n}\right|_{p+q+2},
\end{aligned}
$$

where $M_{5}=\delta M_{4}+\rho^{q+1}$. On the other hand, by (4-9), (4-10) and Corollary A.5 we get

$$
\left|\left(G_{\theta}^{\otimes(n-1-k)}-I^{\otimes(n-1-k)}\right) \omega\right|_{p} \leq \rho(\varepsilon+C) M_{5}^{n-2-k}|\theta||\omega|_{p+q+1} .
$$


In view of (4-5), (4-13) and Corollary A.4, we obtain

$$
\begin{aligned}
\mid\left(I^{\otimes k} \otimes\right. & \left.X \otimes\left(G_{\theta}^{\otimes(n-1-k)}-I^{\otimes(n-1-k)}\right)\right)\left.f_{n}\right|_{p .} \\
& \leq C \rho \cdot \rho(\varepsilon+C) M_{5}^{n-2-k}|\theta| \rho^{n-1-k+(q+2) k} \delta\left|f_{n}\right|_{p+q+2} \\
& =C \delta \rho^{2}(\varepsilon+C) M_{5}^{-1}\left(\rho M_{5}\right)^{n-1-k} \rho^{(q+2) k}|\theta|\left|f_{n}\right|_{p+q+2} \\
& \leq C \delta \rho^{-q+1}(\varepsilon+C)\left(\rho M_{5}\right)^{n-1-k} \rho^{(q+2) k}|\theta|\left|f_{n}\right|_{p+q+2},
\end{aligned}
$$

where we used $M_{5}^{-1}<\rho^{-q-1}$. Therefore we have

$$
\begin{aligned}
& \sum_{k=0}^{n-1}\left|\left(I^{\otimes k} \otimes X \otimes\left(G_{\theta}^{\otimes(n-1-k)}-I^{\otimes(n-1-k)}\right)\right) f_{n}\right|_{p} \\
& \quad \leq C \delta \rho^{-q+1}(\varepsilon+C)\left(\rho M_{5}+\rho^{q+2}\right)^{n-1}|\theta|\left|f_{n}\right|_{p+q+2} .
\end{aligned}
$$

From (4-11), (4-12) and (4-14) we see that

$$
\begin{aligned}
\left|\frac{G_{\theta}^{\otimes n} f_{n}-f_{n}}{\theta}-\gamma_{n}(X) f_{n}\right|_{p} & \leq \varepsilon \rho^{2}\left(\rho M_{5}\right)^{n-1}\left|f_{n}\right|_{p+q+2} \\
& +C \delta \rho^{-q+1}(\varepsilon+C)\left(\rho M_{5}+\rho^{q+2}\right)^{n-1}|\theta|\left|f_{n}\right|_{p+q+2} .
\end{aligned}
$$

Since $\rho M_{5}<\rho M_{5}+\rho^{q+2}<1$ by (4-8), the last quantity is bounded by

$$
\begin{gathered}
\left\{\varepsilon \rho^{2}\left(\rho M_{5}\right)^{-1}+C \delta \rho^{-q+1}(\varepsilon+C)\left(\rho M_{5}+\rho^{q+2}\right)^{-1}|\theta|\right\}\left|f_{n}\right|_{p+q+2} \\
\leq\left(\varepsilon \rho^{-q}+C \delta(\varepsilon+C) \rho^{-2 q-1}|\theta|\right)\left|f_{n}\right|_{p+q+2},
\end{gathered}
$$

where $M_{5}^{-1}<\rho^{-q-1}$ is used again. Since $d \Gamma(X)=\sum_{n=0}^{\infty} \gamma_{n}(X)$, we conclude that

$$
\left\|\frac{\Gamma\left(G_{\theta}\right) \phi-\phi}{\theta}-d \Gamma(X) \phi\right\|_{p} \leq\left(\varepsilon \rho^{-q}+|\theta| C(\varepsilon+C) \delta \rho^{-2 q-1}\right)\|\phi\|_{p+q+2},
$$

whenever $|\theta|<\theta_{0}$. Consequently,

$$
\lim _{\theta \rightarrow 0} \sup _{\|\phi\|_{p+q+2} \leq 1}\left\|\frac{\Gamma\left(G_{\theta}\right) \phi-\phi}{\theta}-d \Gamma(X) \phi\right\|_{p}=0,
$$

which completes the proof.

Q.E.D.

We are now going to a discussion on the infinite rotation group. Following Yoshizawa [23] a linear homeomorphism $g \in G L(E)$ is called a rotation of $E$ if $|g \xi|_{0}=|\xi|_{0}$, i.e., if it can be extended to an orthogonal operator on $H=$ $L^{2}(T, \nu ; \mathbf{R})$. Let $O(E ; H)$ denote the group of all rotations of $E$. Obviously, it is a subgroup of $G L(E)$.

It is noted that $\left(\Gamma,\left(L^{2}\right)\right)$ is a unitary representation of $O(E ; H)$. In fact,

$$
(\Gamma(g) \phi)(x)=\phi\left(g^{*} x\right), \quad \phi \in\left(L^{2}\right), x \in E^{*},
$$


where $g^{*} x$ is defined by

$$
\langle x, g \xi\rangle=\left\langle g^{*} x, \xi\right\rangle, \quad x \in E^{*}, \xi \in E .
$$

Let $U\left((E) ;\left(L^{2}\right)\right)$ be the group of unitary operators on $\left(L^{2}\right)$ which is defined similarly as $O(E ; H)$. It then follows that $\Gamma(g) \in U\left((E) ;\left(L^{2}\right)\right)$ for any $g \in O(E ; H)$.

Let $\left\{G_{\theta}\right\}_{\theta \in \mathbf{R}}$ be a differentiable one-parameter subgroup of $O(E ; H)$ with infinitesimal generator $X$. As is easily seen, $X$ is skew-symmetric in the sense that

$$
\langle X \xi, \eta\rangle=-\langle\xi, X \eta\rangle, \quad \xi, \eta \in E .
$$

Proposition 4.2. Let $X$ be a continuous operator on $E$ which is skew-symmetric in the sense of (4-15). Then there exists a skew-symmetric distribution $\kappa \in E \otimes E^{*}$ such that

$$
d \Gamma(X)=\int_{T \times T} \kappa(s, t)\left(\partial_{s}^{*} \partial_{t}-\partial_{t}^{*} \partial_{s}\right) d s d t
$$

Proof. Consider

$$
\kappa=\frac{1}{2} \sum_{i, j=0}^{\infty}\left\langle e_{\imath}, X e_{j}\right\rangle e_{\imath} \otimes e_{\imath} .
$$

Since $X$ is continuous, there exist $q \geq 0$ and $C>0$ such that $|X \xi|_{0} \leq C|\xi|_{q}$. Hence,

$$
\left|\left\langle e_{i}, X e_{j}\right\rangle\right| \leq\left|e_{i}\right|_{0}\left|X e_{j}\right|_{0} \leq C\left|e_{j}\right|_{q}=C \lambda_{j}^{q}
$$

and $\kappa \in(E \otimes E)^{*}$. Moreover, by a direct calculation, we have

$$
\langle\kappa, \eta \otimes \zeta\rangle=\frac{1}{2}\langle\eta, X \zeta\rangle
$$

This shows that $\kappa \in E \otimes E^{*}$ and that $\kappa$ is skew-symmetric. The right hand side of $(4-15)$ is, therefore, equal to $2 \Xi_{1,1}(\kappa)$ which is a continuous operator on $(E)$ by Theorem 2.6. Since $d \Gamma(X)$ is also continuous, we need only to show that $2 \Xi_{1,1}(\kappa) \phi_{\xi}=d \Gamma(X) \phi_{\xi}$ for exponential vectors $\phi_{\xi} \in(E)$ defined as in (1-9). By (4-3) we have

$$
\left(d \Gamma(X) \phi_{\xi}\right)(x)=\sum_{n=1}^{\infty} \frac{1}{(n-1) !}\left\langle: x^{\otimes n}:,(X \xi) \otimes \xi^{\otimes(n-1)}\right\rangle
$$

and therefore 


$$
\begin{aligned}
\left\langle\left\langle d \Gamma(X) \phi_{\xi}, \phi_{\eta}\right\rangle\right\rangle & =\sum_{n=1}^{\infty} n ! \frac{1}{(n-1) ! n !}\left\langle(X \xi) \otimes \xi^{\otimes(n-1)}, \eta^{\otimes n}\right\rangle \\
& =\sum_{n=1}^{\infty} \frac{1}{(n-1) !}\langle X \xi, \eta\rangle\langle\xi, \eta\rangle^{n-1} \\
& =\langle X \xi, \eta\rangle e^{\langle\xi, \eta\rangle} .
\end{aligned}
$$

On the other hand, in view of Proposition 2.7 and (4-18) we have

$$
2\left\langle\left\langle\Xi_{1,1}(\kappa) \phi_{\xi}, \phi_{\eta}\right\rangle\right\rangle=2\langle\kappa, \eta \otimes \xi\rangle e^{\langle\xi, \eta\rangle}=(\eta, X \xi\rangle e^{\langle\xi, \eta\rangle} .
$$

This completes the proof.

Q.E.D.

In view of Theorem 4.1 and Proposition 4.2 we obtain the following

THEOREM 4.3. Let $X$ be an infinitesimal generator of a regular one-parameter subgroup of $O(E ; H)$. Then, there exists a skew-symmetric distribution $\kappa \in E \otimes E^{*}$ such that

$$
d \Gamma(X)=\int_{T \times T} \kappa(s, t)\left(\partial_{s}^{*} \partial_{t}-\partial_{t}^{*} \partial_{s}\right) d s d t
$$

For a fixed $t \in T$ we define $\Phi_{t} \in(E)^{*}$ by

$$
\left\langle\left\langle\Phi_{t}, \phi\right\rangle=f_{1}(t)\right.
$$

for $\phi \in(E)$ given as in $(4-1)$. It is convenient to use a somewhat formal notation $x(t)=\Phi_{t}(x)$ which is regarded as a coordinate function in white noise calculus. Note that a product $\Phi \phi=\phi \Phi \in(E)^{*}$ is defined for $\Phi \in(E)^{*}$ and $\phi \in(E)$ in a usual manner.

Proposition 4.4. $x(t) \phi(x)=\left(\partial_{t}+\partial_{t}^{*}\right) \phi(x)$ for any $\phi \in(E)$.

The proof is direct, see e.g., [10], [12]. We can thereby regard

$$
\partial_{s}^{*} \partial_{t}-\partial_{t}^{*} \partial_{s}=\left(\partial_{s}^{*}+\partial_{s}\right) \partial_{t}-\left(\partial_{t}^{*}+\partial_{t}\right) \partial_{s}=x(s) \partial_{t}-x(t) \partial_{s}
$$

as a direct analog of an infinitesimal generator of finite dimensional rotations. Therefore Theorem 4.3 is a direct extension of a well-known fact on finite dimensional rotations to the white noise case.

\section{§ 5. Infinite dimensional Laplacians}

We now discuss rotation-invariance of infinite dimensional Laplacians as a 
simple application of a general theory established in the previous sections.

The distribution $\tau \in(E \otimes E)^{*}$ is already defined in (1-4) and, in view of Theorem 2.6, we see that

$$
\Xi_{0,2}(\tau)=\int_{T \times T} \tau(s, t) \partial_{s} \partial_{t} d s d t=\Delta_{G}
$$

becomes a continuous operator on $(E)$. On the other hand, note that $\tau \in E \otimes E^{*}$. In fact, since $\langle\tau, \eta \otimes \zeta\rangle=\langle\eta, \zeta\rangle$ for $\eta, \zeta \in E$, the corresponding operator in $\mathscr{L}(E)$ is nothing but the identity (see $(2-10)$ ). Hence, using Theorem 2.6 again, we observe that

$$
\Xi_{1,1}(\tau)=\int_{T \times T} \tau(s, t) \partial_{s}^{*} \partial_{t} d s d t=N
$$

is also a continuous operator on $(E)$. These operators are called the Gross Laplacian and the number operator, respectively. Note that $\Delta_{B}=-N$ is often called the Beltrami Laplacian, see e.g. [12]. In fact, with the help of Proposition 2.7, for an exponential vector $\phi_{\xi}, \xi \in E_{\mathbf{C}}$, we obtain

$$
\begin{aligned}
\Xi_{0,2}(\tau) \phi_{\xi}(x) & =\sum_{n=0}^{\infty} \frac{1}{n !}\left\langle: x^{\otimes n}:\langle\tau, \xi \otimes \xi\rangle \xi^{\otimes n}\right\rangle \\
& =\langle\xi, \xi\rangle \phi_{\xi}(x)=\Delta_{G} \phi_{\xi}(x)
\end{aligned}
$$

and

$$
\begin{aligned}
\Xi_{1,1}(\tau) \phi_{\xi}(x) & =\sum_{n=0}^{\infty} \frac{1}{n !}\left\langle: x^{\otimes(n+1)}:, \xi^{\otimes(n+1)}\right\rangle \\
& =\sum_{n=0}^{\infty} n\left\langle: x^{\otimes n}: \frac{\xi^{\otimes n}}{n !}\right\rangle=N \phi_{\xi} .
\end{aligned}
$$

In this section we characterize $\Delta_{G}$ and $N$ among quadratic forms of operators $\partial_{t}$ and $\partial_{t}^{*}$ in terms of rotation invariance. The main assertions are the following.

THEOREM 5.1. If

$$
\Xi_{0,2}(\lambda)=\int_{T \times T} \lambda(s, t) \partial_{s} \partial_{t} d s d t . \quad \lambda \in\left(E_{\mathbf{C}} \otimes E_{\mathbf{C}}\right)^{*}
$$

is invariant under $O(E ; H)$, then it is a constant multiple of the Gross Laplacian.

THEOREM 5.2. If 


$$
\Xi_{1,1}(\lambda)=\int_{T \times T} \lambda(s, t) \partial_{s}^{*} \partial_{t} d s d t, \quad \lambda \in E_{\mathbf{C}} \otimes E_{\mathbf{C}}^{*},
$$

is invariant under $O(E ; H)$, then it is a constant multiple of the number operator.

First note that if a continuous operator $\Xi$ on $(E)$ is invariant under $O(E ; H)$ then

$$
[\Xi, d \Gamma(X)]=0
$$

for any infinitesimal genarator $X$ of a regular one-parameter subgroup $\left\{G_{\theta}\right\}_{\theta \in \mathbf{R}} \subset$ $O(E ; H)$. In fact, with the help of Theorem 4.1 one can differentiate at $\theta=0$ the identity $\Gamma\left(G_{\theta}\right) \Xi \phi=\Xi \Gamma\left(G_{\theta}\right) \phi, \phi \in(E)$, to obtain (5-1).

Lemma 5.3. Let $\lambda \in\left(E_{\mathbf{C}} \otimes E_{\mathbf{C}}\right)^{*}$ and $X$ an infinitesimal generator of a regular one-parameter subgroup of $O(E ; H)$. Then, for an exponential vector $\phi_{\xi}$, $\xi \in E_{\mathbf{C}}$, we have

$$
\left[\Xi_{0,2}(\lambda), d \Gamma(X)\right] \phi_{\xi}=2\langle\hat{\lambda}, X \xi \otimes \xi\rangle \phi_{\xi},
$$

where $\hat{\lambda}$ is the symmetrization of $\lambda$.

Lemma 5.4. Let $\lambda \in E_{\mathbf{C}} \otimes E_{\mathbf{C}}^{*}$ and $X$ an infinitesimal generator of a regular one-parameter subgroup of $O(E ; H)$. Then, we have

$$
\left[\Xi_{1,1}(\lambda), d \Gamma(X)\right]=-d \Gamma([X, L]),
$$

where $L$ is a continuous operator on $E_{\mathbf{C}}$ defined by $\langle\lambda, \xi \otimes \eta\rangle=\langle\xi, L \eta\rangle$, $\xi, \eta \in E_{\mathbf{C}}$.

First we note that $d \Gamma(X)=2 \Xi_{1,1}(\kappa)$, where $\kappa \in E \otimes E^{*}$ is given as in (4-15). Then, for the proofs of the above lemmas we need only to apply Proposition 2.7. It is noteworthy that $\Xi_{1,1}(\lambda)=d \Gamma(L)$ for $\lambda$ and $L$ being the same as in Lemma 5.4 .

Proof of Theorem 5.1. Suppose that $\Xi_{0,2}(\lambda)$ is invariant under $O(E ; H)$. It then follows from Lemma 5.3 that

$$
\langle\hat{\lambda}, X \xi \otimes \xi\rangle=0, \quad \xi \in E_{\mathbf{C}},
$$

for any infinitesimal generator of a regular one-parameter subgroup of $O(E ; H)$. Suppose that $i \neq j$ are arbitrarily fixed non-negative integers and define $X$ as 


$$
\left\{\begin{array}{l}
X e_{i}=e_{j} \\
X e_{j}=-e_{i} \\
X e_{k}=0, \quad k \neq i, j .
\end{array}\right.
$$

Then we obtain

$$
0=\left\langle\hat{\lambda}, X e_{i} \otimes e_{i}\right\rangle=\left\langle\hat{\lambda}, e_{j} \otimes e_{i}\right\rangle
$$

and

$$
\begin{aligned}
0=\left\langle\hat{\lambda}, X\left(e_{i}+e_{j}\right) \otimes\left(e_{i}+e_{j}\right)\right\rangle & =\left\langle\hat{\lambda}, X e_{i} \otimes e_{j}+X e_{j} \otimes e_{i}\right\rangle \\
& =\left\langle\hat{\lambda}, e_{j} \otimes e_{j}\right\rangle-\left\langle\hat{\lambda}, e_{i} \otimes e_{i}\right\rangle .
\end{aligned}
$$

Hence $\left\langle\hat{\lambda}, e_{1} \otimes e_{i}\right\rangle=c$ is independent of $i=0,1,2, \ldots$ and $\left\langle\hat{\lambda}, e_{i} \otimes e_{j}\right\rangle=0$ for $i \neq j$. Therefore $\hat{\lambda}=c \tau$ and we conclude that $\Xi_{0,2}(\lambda)=\Xi_{0,2}(\hat{\lambda})=\Xi_{0,2}(c \tau)=c \Delta_{G}$.

Q.E.D.

Proof of Theorem 5.2. Suppose that $\Xi_{1,1}(\lambda)$ is invariant under $O(E ; H)$. It then follows from Lemma 5.4 that $d \Gamma([X, L])=0$, and therefore $[X, L]=0$. Let $X$ be the same as in $(5-2)$. Then, for $k \neq i, j$ we have

$$
\begin{aligned}
0=L X e_{k}=X L e_{k} & =\sum_{l=0}^{\infty}\left\langle L e_{k}, e_{l}\right\rangle X e_{l} \\
& =\left\langle L e_{k}, e_{i}\right\rangle X e_{\imath}+\left\langle L e_{k}, e_{j}\right\rangle X e_{j} \\
& =\left\langle L e_{k}, e_{\imath}\right\rangle e_{j}-\left\langle L e_{k}, e_{\jmath}\right\rangle e_{i} .
\end{aligned}
$$

Therefore, $\left\langle L e_{k}, e_{i}\right\rangle=\left\langle L e_{k}, e_{\jmath}\right\rangle=0$. In other words,

$$
\left\langle\lambda, e_{\imath} \otimes e_{j}\right\rangle=\left\langle e_{i}, L e_{j}\right\rangle=0 \quad i \neq j .
$$

On the other hand,

$$
\begin{aligned}
\left\langle L e_{i}, e_{i}\right\rangle & =-\left\langle L X e_{j}, e_{i}\right\rangle=-\left\langle X L e_{j}, e_{i}\right\rangle \\
& =\left\langle L e_{j}, X e_{i}\right\rangle=\left\langle L e_{j}, e_{j}\right\rangle .
\end{aligned}
$$

Namely, $\left\langle\lambda, e_{i} \otimes e_{\imath}\right\rangle=\left\langle e_{\imath}, L e_{i}\right\rangle=c$ is independent of $i=0,1,2, \ldots$ Therefore $\lambda$ $=c \tau$ and $\Xi_{1,1}(\lambda)=\Xi_{1,1}(c \tau)=c N$.

Remark. During the above discussion we used only a subgroup of $O(E ; H)$ consisting of rotations which act identically on the subspace spanned by $\left\{e_{n}, e_{n+1}, \ldots\right\}$ for some $n=0,1,2, \ldots$ This group is sometimes denoted by $O_{\infty}$ and is an inductive limit of $O(n)$. It is also interesting to consider another subgroups, for example, a group of transformations of $T$ which is naturally imbedded 
in $O(E ; H)$. In general, a one-parameter subgroup of $O(E ; H)$ arising from transformations on $T$ is called a whisker and plays an interesting role in a study of symmetry of Brownian motion, in this connection see [4], [14], [23].

\section{Appendix. Some inequalities}

Proposition A.1. Let $K \in \mathscr{L}\left(E_{\mathbf{C}}^{\otimes m}, E_{\mathbf{C}}^{\otimes l}\right)$ such that $|K \eta|_{p} \leq C|\eta|_{p+q}, \eta \in$ $E_{\mathrm{C}}^{\otimes m}$, for some $p, q \geq 0$ and $C \geq 0$. Then, for any $n \geq 0$,

$$
\left|\left(K \otimes I^{\otimes n}\right) f_{m+n}\right|_{p} \leq C \rho^{q n}\left|f_{m+n}\right|_{p+q}, \quad f_{m+n} \in E_{\mathbf{C}}^{\otimes(m+n)} .
$$

Proof. By Fourier expansion we have

$$
f_{m+n}=\sum_{i_{1}, \ldots, i_{n}=0}^{\infty} g_{i_{1}, \ldots, i_{n}} \otimes e_{i_{1}} \otimes \cdots \otimes e_{i_{n}},
$$

where $g_{i_{1}, \ldots, i_{n}} \in E_{\mathbf{C}}^{\otimes m}$ and

$$
\left|f_{m+n}\right|_{r}^{2}=\sum_{i_{1}, \ldots, i_{n}=0}^{\infty} \lambda_{i_{1}}^{2 r} \cdots \lambda_{i_{n}}^{2 r}\left|g_{i_{1}, \ldots, i_{n}}\right|_{r}^{2}, \quad r \geq 0 .
$$

Then,

$$
\begin{aligned}
\left|\left(K \otimes I^{\otimes n}\right) f_{m+n}\right|_{p}^{2} & =\left|\sum K g_{i_{1}, \ldots, i_{n}} \otimes e_{i_{1}} \otimes \cdots \otimes e_{i_{n}}\right|_{p}^{2} \\
& =\sum \lambda_{i_{1}}^{2 p} \cdots \lambda_{i_{n}}^{2 p}\left|K g_{i_{1}, \ldots, i_{n}}\right|_{p}^{2} \\
& \leq \sum \lambda_{i_{1}}^{2 p} \cdots \lambda_{i_{n}}^{2 p} C^{2}\left|g_{i_{1}, \ldots, i_{n}}\right|_{p+q}^{2} \\
& =C^{2} \sum \lambda_{i_{1}}^{2 q} \cdots \lambda_{i_{n}}^{2 q} \lambda_{i_{1}}^{2(p+q)} \cdots \lambda_{i_{n}}^{2(p+q)}\left|g_{i_{1}, \ldots, i_{n}}\right|_{p+q}^{2} \\
& \leq C^{2} \rho^{2 q n}\left|f_{m+n}\right|_{p+q}^{2},
\end{aligned}
$$

where we used $1<\rho^{-1}=\lambda_{0} \leq \lambda_{1} \leq \lambda_{2} \leq \cdots$.

Q.E.D.

COROLlary A.2. For $f_{m+n} \in E_{\mathbf{C}}^{\otimes(m+n)}$ and $p \geq 0$, we have

$$
\left|\left(\left(A^{p}\right)^{\otimes m} \otimes I^{\otimes n}\right) f_{m+n}\right|_{0} \leq \rho^{p n}\left|f_{m+n}\right|_{p .}
$$

Proposition A.3. For $i=1,2, \ldots, d$, let $K_{i} \in \mathscr{L}\left(E_{\mathbf{C}}^{\otimes m_{i}}, E_{\mathbf{C}}^{\otimes l_{i}}\right)$. Assume that $\left|K_{i} \xi_{i}\right|_{p} \leq C_{i}\left|\xi_{i}\right|_{q}, \xi_{i} \in E_{\mathbf{C}}^{\otimes m_{i}}$,for some $p, q \geq 0$ and $C_{i} \geq 0$. Then, for any $i$ we have

$$
\left|\left(K_{1} \otimes \cdots \otimes K_{d}\right) \omega\right|_{p} \leq C_{1} \cdots C_{d} \rho^{m_{i}} \delta^{m-m_{i}}|\omega|_{q+1}, \quad \omega \in E_{\mathbf{C}}^{\otimes m},
$$


where $m=m_{1}+\cdots+m_{d}$.

Remark. Putting $m_{i}=\max \left(m_{1}, \cdots, m_{d}\right)$, we obtain the best estimate. Since $\rho<\delta$, we have

$$
\left|\left(K_{1} \otimes \cdots \otimes K_{d}\right) \omega\right|_{p} \leq C_{1} \cdots C_{d} \delta^{m}|\omega|_{q+1} . \quad \omega \in E_{\mathbf{C}}^{\otimes m} .
$$

This is also useful.

Proof. It is sufficient to prove the inequality for $i=1$. Let $\mathscr{B}_{i}$ be the basis of $E_{\mathbf{C}}^{\otimes m_{i}}$, namely,

$$
\mathscr{B}_{i}=\left\{f_{i}=e_{\jmath(1)} \otimes \cdots \otimes e_{j\left(m_{i}\right)} ; j(1), \cdots, j\left(m_{i}\right) \geq 0\right\} .
$$

Then, each $\omega \in E_{\mathbf{C}}^{\otimes m}$ is expressed as

$$
\omega=\sum_{\substack{f_{i} \in \mathscr{B}_{i} \\ 2 \leq i \leq d}} g\left(f_{2}, \cdots, f_{d}\right) \otimes f_{2} \otimes \cdots \otimes f_{d},
$$

where $g\left(f_{2}, \cdots, f_{d}\right) \in E_{\mathbf{C}}^{\otimes m_{1}}$ and

$$
|\omega|_{r}^{2}=\sum\left|g\left(f_{2}, \ldots, f_{d}\right)\right|_{r}^{2}\left|f_{2}\right|_{r}^{2} \cdots\left|f_{d}\right|_{r}^{2}
$$

Then, using the Schwarz inequality, we obtain

$$
\begin{aligned}
\mid & \left.\left(K_{1} \otimes \cdots \otimes K_{d}\right) \omega\right|_{p} ^{2} \leq\left(\sum\left|K_{1} g\left(f_{2}, \ldots, f_{d}\right)\right|_{p}\left|K_{2} f_{2}\right|_{p} \cdots\left|K_{d} f_{d}\right|_{p}\right)^{2} \\
\leq & C_{1}^{2} \cdots C_{d}^{2}\left(\sum\left|g\left(f_{2}, \ldots, f_{d}\right)\right|_{q}\left|f_{2}\right|_{q} \cdots\left|f_{d}\right|_{q}\right)^{2} \\
\leq & C_{1}^{2} \cdots C_{d}^{2}\left(\sum\left|g\left(f_{2}, \cdots, f_{d}\right)\right|_{q+1}^{2}\left|f_{2}\right|_{q+1}^{2} \cdots\left|f_{d}\right|_{q+1}^{2}\right) \\
& \times\left(\sum\left|g\left(f_{2}, \ldots, f_{d}\right)\right|_{q+1}^{-2}\left|g\left(f_{2}, \ldots, f_{d}\right)\right|_{q}^{2}\left|f_{2}\right|_{q+1}^{-2}\left|f_{2}\right|_{q}^{2} \cdots\left|f_{d}\right|_{q+1}^{-2}\left|f_{d}\right|_{q}^{2}\right) .
\end{aligned}
$$

Since $\left|g\left(f_{2}, \ldots, f_{d}\right)\right|_{q} \leq \rho^{m_{1}}\left|g\left(f_{2}, \ldots, f_{d}\right)\right|_{q+1}$, we obtain

$$
\left|\left(K_{1} \otimes \cdots \otimes K_{d}\right) \omega\right|_{p}^{2} \leq C_{1}^{2} \cdots C_{d}^{2}|\omega|_{q+1}^{2} \rho^{2 m_{1}} \prod_{i=2}^{d} \sum_{f_{i} \in \mathscr{B}_{i}}\left(\frac{\left|f_{i}\right|_{q}}{\left|f_{i}\right|_{q+1}}\right)^{2} .
$$

If $f_{\imath}=e_{j(1)} \otimes \cdots \otimes e_{j\left(m_{i}\right)}$, we have $\left|f_{i}\right|_{q}=\left(\lambda_{j(1)} \cdots \lambda_{j\left(m_{i}\right)}\right)^{q}$, and therefore

$$
\sum_{f_{i} \in \mathscr{B}_{i}}\left(\frac{\left|f_{i}\right|_{q}}{\left|f_{i}\right|_{q+1}}\right)^{2}=\sum_{j(1), \ldots, j\left(m_{i}\right)=0}^{\infty}\left(\lambda_{j(1)} \cdots \lambda_{j(m i)}\right)^{-2}=\left(\sum_{j=0}^{\infty} \lambda_{j}^{-2}\right)^{m_{i}}=\delta^{2 m_{i}} .
$$

Consequently,

$$
\left|\left(K_{1} \otimes \cdots \otimes K_{d}\right) \omega\right|_{p} \leq C_{1} \cdots C_{d}|\omega|_{q+1} \rho^{m_{1}} \delta^{m_{2}+\cdots+m_{d}} .
$$

This proves the assertion.

Q.E.D. 
Corollary A.4. For $i=1,2, \ldots, d$, let $K_{i} \in \mathscr{L}\left(E_{\mathbf{C}}^{\otimes m_{i}}, E_{\mathbf{C}}^{\otimes l_{i}}\right)$. Assume that $\left|K_{i} \xi_{i}\right|_{p} \leq C_{\imath}\left|\xi_{i}\right|_{p+q}, \xi_{i} \in E_{\mathbf{C}}^{\otimes m_{i}}$, for some $p, q \geq 0$ and $C_{i} \geq 0$. Then, for any $i$ we have

$$
\left|\left(I^{\otimes k} \otimes K_{1} \otimes \cdots \otimes K_{d}\right) \omega\right|_{p} \leq C_{1} \cdots C_{d} \rho^{m_{i+1}(q+1) k} \delta^{m-m i}|\omega|_{p+q+1}, \quad \omega \in E_{\mathbf{C}}^{\otimes(k+m)},
$$

where $m=m_{1}+\cdots+m_{d}$.

Proof. Immediate from Propositions A.1 and A.3.

Q.E.D.

Corollary A.5. Let $B \in \mathscr{L}\left(E_{\mathbf{C}}\right)$ be such that $|B \xi|_{p} \leq C_{1}|\xi|_{p+q}$ and $|(B-I) \xi|_{p} \leq C_{2}|\xi|_{p+q}$. Then,

$$
\left|\left(B^{\otimes n}-I^{\otimes n}\right) f_{n}\right|_{p} \leq \rho C_{2}\left(\delta C_{1}+\rho^{q+1}\right)^{n-1}|f|_{p+q+1}, \quad f_{n} \in E_{\mathbf{C}}^{\otimes n} .
$$

Proof. We need only simple calculation and Corollary A.4.

$$
\begin{aligned}
\left|\left(B^{\otimes n}-I^{\otimes n}\right) f_{n}\right|_{p} & \left.\leq \sum_{k=0}^{n-1} \mid B^{\otimes(n-1-k)} \otimes(B-I) \otimes I^{\otimes k}\right)\left.f_{n}\right|_{p} \\
& \leq \sum_{k=0}^{n-1} C_{1}^{n-1-k} C_{2} \rho^{1+(q+1) k} \delta^{n-1-k}\left|f_{n}\right|_{p+q+1} \\
& \leq \rho C_{2}\left(\delta C_{1}+\rho^{q+1}\right)^{n-1}\left|f_{n}\right|_{p+q+1} .
\end{aligned}
$$

This completes the proof.

Q.E.D.

\section{REFERENCES}

[1] Yu. M. Berezansky and Yu. G. Kondrat'ev, "Spectral Methods in Infinite Dimensional Analysis," (in Russian), Kiev, 1988.

[2] T. Hida, "Analysis of Brownian Functionals," Carleton Math. Lect. Notes Vol. 13, Carleton University, Ottawa, 1975.

[ 3 ] T. Hida, "Brownian Motion," Springer-Verlag, 1980.

[4] T. Hida, I. Kubo, H. Nomoto and H. Yoshizawa, On projective invariance of Brownian Motion, Publ. RIMS, Kyoto Univ. Ser. A., 4 (1969), 595-609.

[ 5 ] T. Hida, H.-H. Kuo, J. Potthoff and L. Streit, "White Noise: An Infinite Dimensional Calculus," Monograph in preparation.

[6] T. Hida, K.-S. Lee and S.-S. Lee, Conformal invariance of white noise, Nagoya Math. J., 98 (1985), 87-98.

[7] T. Hida and J. Potthoff, White noise analysis-An overview, in "White Noise Analysis (T. Hida et al. Eds.)," World Scientific, Singapore/New Jersey/London/Hong Kong, 1990, pp. 140-165.

[8] T. Hida and K. Saitô, White noise analysis and the Lévy Laplacian, in "Stochastic Processes in Physics and Engineering (S. Albeverio et al. Eds.)," D. Reidel Pub., Dordrecht/Boston/Lancaster/Tokyo, 1988, pp. 177-184. 
)] P. Krée, La théorie des distributions en dimension quelconque et l'intégration stochastique, in "Stochastic Analysis and Related Topics (H. Korezlioglu and A. S. Ustunel Eds.)," Lect. Notes in Math. Vol. 1316, Springer-Verlag, 1988, pp. $170-233$.

0] I. Kubo and S. Takenaka, Calculus on Gaussian white noise I-IV, Proc. Japan Acad., 56A (1980), 376-380; 411-416; 57A (1981), 433-437; 58A (1982), $186-189$.

1] I. Kubo and Y. Yokoi, A remark on the space of testing random variables in the white noise calculus, Nagoya Math. J., 115 (1989), 139-149.

2] H.-H. Kuo, On Laplacian operators of generalized Brownian functionals, in "Stochastic Processes and Their Applications (K. Itô and T. Hida Eds.)," Lect. Notes in Math. Vol. 1203, Springer-Verlag, 1986, pp. 119-128.

3] H.-H. Kuo, N. Obata and K. Saitô, Lévy Laplacian of generalized functions on a nuclear space, J. Funct. Anal., 94 (1990), 74-92.

4] K.-S. Lee, White noise approach to Gaussian random field, Nagoya Math. J., 119 (1990), 93-106.

5] P. Lévy, "Problèmes Concrets d'Analyse Fonctionnelle," Gauthier-Villars, Paris, 1951.

6] P. A. Meyer, Distributions, noyaux, symboles d'après Krée, in "Séminaire de Probabilités XXII (J. Azéma et al. Eds.)," Lect. Notes in Math. Vol. 1321, Springer-Verlag, 1988, pp. 467-476.

7] N. Obata, A characterization of the Lévy Laplacian in terms of infinite dimensional rotation groups, Nagoya Math. J., 118 (1990), 111-132.

8] J. Potthoff and J.-A. Yan, Some results about test and generalized functionals of white noise, in "Probability Theory (L. H. Y. Chen et al. Eds.)," Walter de Gruyter, Berlin/New York, 1992, pp. 121-145.

9] K. Saitô, Itô's formula and Lévy's Laplacian, Nagoya Math. J., 108 (1987), 67-76; II, ibid., 123 (1991), 153-169.

0] F. Treves, "Topological Vector Spaces, Distributions and Kernels," Academic Press, New York/London, 1967.

1] J.-A. Yan, Products and transforms of white noise functionals, preprint (1990).

2] Y. Yokoi, Positive generalized white noise functionals, Hiroshima Math. J., 20 (1990), 137-157.

3] H. Yoshizawa, Rotation group of Hilbert space and its application to Brownian motion, in "Proc. International Conference on Functional Analysis and Related Topics," University of Tokyo Press, Tokyo, 1970, pp. 414-423.

Hida and K. Saitô

epartment of Mathematics

'eijo University

agoya 468, Japan

- Obata

epartment of Mathematics

shool of Science

agoya University

agoya 464-01, Japan 
